\title{
IUFOST2006/1025 \\ Lessons learnt in development and application of detection methods for foodborne parasites
}

\author{
H.V. Smith ${ }^{\mathrm{a}}$ and N. Cook ${ }^{\mathrm{b}}$ \\ ${ }^{a}$ Scottish Parasite Diagnostic Laboratory, Stobhill Hospital, G21 3UW Glasgow, United Kingdom \\ ${ }^{b}$ DEFRA Central Science Laboratory, Sand Hutton, YO1 1LZ York, United Kingdom \\ huw.smith@northglasgow.scot.nhs.uk
}

Increased demand, global sourcing, and rapid transport of soft fruit and salad vegetables can enhance both the likelihood of surface contamination and survival of the transmissive stages of protozoa pathogenic to man. Given the low infectious doses [9 to 100 (oo)cysts of Cryptosporidium, Cyclospora and Giardia], surface contamination with visually undetectable amounts of viable parasites, in produce which receives minimal washing prior to ingestion, poses a threat to public health. Foodborne outbreaks of cryptosporidiosis, cyclosporiasis and giardiasis have been documented both in developed and developing countries.

Standardised methods are necessary for detecting protozoan parasites on foods. We report on optimizing and validating methods for detecting Cryptosporidium parvum on lettuce and raspberries. These methods are based on four basic stages: extraction of oocysts from the foodstuffs, concentration of the extract and separation of the oocysts from food materials, staining of the oocysts to allow their visualization, and identification of oocysts by microscopy. The method to detect C. parvum on raspberries recovered $41.0 \pm 13.0 \%(\mathrm{n}=30)$ of artificially contaminated oocysts.

For lettuce artificially contaminated at low (8.5 - 14.2), medium (53.5 - 62.6), and high (111.3 - 135.0) oocyst levels, the results of interlaboratory collaborative trials involved eight expert UK laboratories revealed an overall sensitivity (correct identification of all artificially contaminated lettuce samples) of $89.6 \%$, and a specificity (correct identification of non-contaminated samples) of $85.4 \%$. The total median percentage recovery (from all artificially contaminated samples) produced by the method was $30.4 \%$, and the method was just as reproducible between laboratories, as repeatable within a laboratory. For raspberry samples artificially contaminated at low $(8.5-26.8)$, medium $(29.7-65.7)$, and high $(53.9-131.3)$ oocyst levels, the method had an overall sensitivity of $95.8 \%$, and a specificity of $83.3 \%$. The total median percentage recovery produced by the method was $44.3 \%$. The method was just as reproducible between laboratories, as repeatable within a laboratory. The results of the collaborative trial indicate that these assays can be used effectively in analytical microbiological laboratories. 